\title{
Retraction Note: On projection-invariant submodules of QTAG-modules
}

Fahad Sikander $^{1 *}$, Alveera Mehdi ${ }^{2}$ and Sabah A. R. K. Naji ${ }^{3}$

* Correspondence: f.sikander@seu. edu.sa

${ }^{1}$ College of Computation and Informatics, Saudi Electronic University, Jeddah 23442, Saudi Arabia

\section{Retraction Note: J Egypt Math Soc} https://doi.org/10.1016/j.joems.2015.01.005

The Editor-in-Chief has retracted this article [1] because the results have been previously published by Danchev and Goldsmith [2]. This article is therefore redundant.

Fahad Sikander does not agree to this retraction. Alveera Mehdi and Sabah A.R.K. Naji have not responded to correspondence from the Publisher about this retraction.

\section{Author details}

${ }^{1}$ College of Computation and Informatics, Saudi Electronic University, Jeddah 23442, Saudi Arabia. ${ }^{2}$ Department of Mathematics, Aligarh Muslim University, Aligarh 202 002, India. ${ }^{3}$ Department of Mathematics, Al-Bayda University, Al-Bayda, Yemen.

Published online: 29 October 2019
References
1. Sikander, F., Mehdi, A., Naji, S.A.: On projection-invariant submodules of QTAG-modules. J. Egypt. Math. Soc. 24(2), 156- 159 (2016)
2. Danchev, P., Goldsmith, B.: On projection-invariant subgroups of Abelian p-groups. Contemp. Math. 576, 31-40 (2012) 